\title{
Langerhans-type and monocyte-derived human dendritic cells have different susceptibilities to mRNA electroporation with distinct effects on maturation and activation: implications for immunogenicity in dendritic cell-based immunotherapy
}

David J Chung ${ }^{1,3,4,6,7^{*}}$, Emanuela Romano ${ }^{1,6,8}$, Katherine B Pronschinske ${ }^{1,6}$, Justin A Shyer ${ }^{1,6}$, Milena Mennecozzi ${ }^{2,6,9}$, Erin T St Angelo ${ }^{1,6}$ and James W Young ${ }^{1,3,4,5,6,7}$

\begin{abstract}
Background: mRNA electroporation of dendritic cells (DCs) facilitates processing and presentation of multiple peptides derived from whole antigen, tailored to different HLA molecules. Clinical responses to electroporated moDC vaccines, however, have been suboptimal. Human Langerhans-type DCs (LCs) are the most potent conventional DC subtype for inducing CD8 ${ }^{+}$cytotoxic T lymphocytes (CTLS) in vitro. We recently demonstrated that Wilms' tumor 1 (WT1) mRNA-electroporated LCs are superior to moDCs as stimulators of tumor antigen-specific $\mathrm{CD}^{+}$CTLs, even though they are comparable stimulators of allogeneic $\mathrm{T}$ cell proliferative responses. A detailed comparative evaluation of the effects of mRNA electroporation on LCs versus moDCs, however, is needed.

Methods: Immature and partially-matured human moDCs and LCs electroporated with mRNA were compared for transfection efficiency, phenotypic changes, viability, retention of transgene expression after cryopreservation, and immunogenicity. Student t test was used for each pairwise comparison. One-way analysis of variance was used for multiple group comparisons.

Results: Transfection efficiency after electroporation with enhanced green fluorescent protein (eGFP) mRNA was higher for immature than for partially-matured moDCs. In contrast, transfection efficiency was higher for partiallymatured than for immature LCs, with the additional benefit that electroporation itself increased maturation and activation of $C D 83^{+} H L A-D R^{\text {bright }}$ LCs but not moDCs. Electroporation did not impair final maturation and activation of either DC subtype, after which both mRNA-electroporated LCs and moDCs were functionally similar in stimulating allogeneic T cell proliferation, a standard assay of DC immunogenicity.
\end{abstract}

Conclusions: These findings support mRNA electroporation of DCs, and in particular LCs, as an effective non-viral method to stimulate specific, potent $C D 8^{+} C T L$ responses. The differences between $L C s$ and moDCs regarding this form of antigen-loading have important implications for DC-based immunotherapies.

Keywords: mRNA electroporation, Dendritic cells, Cancer, Immunotherapy

\footnotetext{
* Correspondence: chungd1@mskcc.org

'Laboratory of Cellular Immunobiology, New York, NY, USA

${ }^{3}$ Adult Bone Marrow Transplant Service, Division of Hematologic Oncology,

New York, NY, USA

Full list of author information is available at the end of the article
} 


\section{Introduction}

Effective therapeutic cancer vaccination requires the optimization of tumor antigen presentation by antigenpresenting cells (APCs) to induce strong antigen-specific $\mathrm{T}$ cell responses, especially $\mathrm{CD} 8^{+}$cytolytic $\mathrm{T}$ lymphocytes (CTLs) with a sufficiently broad repertoire and immunologic memory [1]. Human conventional dendritic cells (DCs) are the most potent APCs and are critical to the onset of immunity. DCs prime $\mathrm{T}$ cell responses by coupling antigen to all the requisite co-stimulatory, cytokine, and chemokine signals required for the activation of naive and resting $\mathrm{T}$ cells [2]. Numerous studies have demonstrated the feasibility of DC-based immunization to induce host responses against tumors [3].

Electroporation of DCs with mRNA encoding specific tumor-associated antigens is an effective non-viral method to stimulate $\mathrm{T}$ cell responses in vitro and in vivo [4-11]. This method of antigen loading, which facilitates processing and presentation of multiple class I and II MHC-restricted epitopes from the translated protein [12], is more efficient than peptide pulsing and less problematic than retroviral transgenes, which carry the risk of genome integration [13]. mRNA electroporation also allows individuals of any HLA type to process and present peptides tailored to their own MHC molecules.

Monocyte-derived DCs (moDCs) are the most commonly used DC subtype in cancer vaccines, and the induction of tumor-specific CTLs in vitro by mRNAtransfected moDCs has been reported in several studies [4-10]. Clinical responses to moDC-based vaccines, however, have not always achieved optimal stimulation of antigen-specific CTLs; and data on clinical responses to vaccination with mRNA-electroporated moDCs are limited [7,11].

Human Langerhans-type DCs (LCs) derived from $\mathrm{CD} 4^{+}$hematopoietic progenitor cells are the most potent conventional DC subtype for stimulating $\mathrm{CD}^{+}$ CTLs in vitro [14-17]. We recently showed that WT1 mRNA-electroporated LCs are superior to moDCs as stimulators of antigen-specific CTLs in vitro, using an IL15R- $\alpha /$ IL15/pSTAT5-dependent mechanism [16]. LCs synthesize abundant IL15 mRNA and protein, whereas moDCs are dependent on exogenous IL15 for stimulating comparably potent WT1-specific CTLs [16]. The effects of mRNA electroporation on moDCs have been described [18]. A detailed comparative evaluation of the effects of mRNA electroporation on LCs versus moDCs is still needed, however.

In this study, we compared moDCs and LCs after mRNA electroporation for transfection efficiency, phenotypic changes, viability, retention of transgene expression after cryopreservation, and allo-stimulatory capacity. Our findings clearly demonstrate that the maturation state of moDCs and LCs differentially affects their susceptibility to electroporation, and electroporation itself has a useful maturational effect on LCs but not moDCs. These findings underscore the importance of tailoring electroporation conditions to specific DC subtypes when designing DC-based immunotherapies.

\section{Methods}

\section{Blood samples}

Peripheral blood mononuclear cells (PBMC) or granulocyte colony stimulating factor (G-CSF)-elicited CD34 $4^{+}$ hematopoietic progenitor cells (HPC) were obtained from healthy volunteers or allogeneic hematopoietic stem cell transplant donors. Buffy coats purchased from the Greater New York Blood Center, American Red Cross, were also used as a source of cells from healthy donors. Biospecimen sample collection and use adhered to protocols approved by the Institutional Review and Privacy Board of Memorial Hospital, Memorial SloanKettering Cancer Center (MSKCC).

\section{Media, serum, and non-cytokine reagents}

For moDC cultures, complete RPMI 1640 was supplemented with $10 \mathrm{mM}$ HEPES (N-2-hydroxyethylpiperazineN'-2-ethanesulfonic acid), $1 \%$ penicillin/streptomycin (Media Laboratory, MSKCC), $50 \mu \mathrm{M}$ 2-mercaptoethanol (GibcoBRL Life Technologies), $2 \mathrm{mM}$ L-glutamine (GibcoBRL), and heat-inactivated, autologous singledonor or pooled human serum ( $1 \%$ or $10 \% \mathrm{vol} / \mathrm{vol})$. For LC cultures, X-VIVO 15 (BioWhittaker) was only supplemented with cytokines (see below). All media and reagents were endotoxin-free.

\section{Generation of moDCs and LCs with recombinant human cytokines}

MoDCs were generated from PBMCs, and LCs were generated from G-CSF-elicited CD34 ${ }^{+}$HPCs. Media, media supplements, and cytokines were exactly as published [14]. In brief, for immature moDC generation, tissue culture plastic adherent $\mathrm{CD}_{1} 4^{+}$monocytes were cultured in complete RPMI-1\% normal human serum (NHS) with GM-CSF and IL-4 for 5 to 6 days. For immature LC generation, CD34 ${ }^{+}$ HPCs were cultured in serum-free X-VIVO 15 , supplemented with GM-CSF, TGF- $\beta$, and TNF- $\alpha$, to which $c$-kit-ligand and FLT-3-ligand were added for only the first 5 to 6 days of a 10- to 12-day culture. Terminal maturation of moDCs and LCs was induced with a combination of TNF- $\alpha$, IL-1 $\beta$, IL-6, and prostaglandin $\mathrm{E}_{2}$.

\section{T lymphocytes}

$\mathrm{T}$ cells were obtained from tissue culture plasticnonadherent PBMCs, further purified by nonadherence and elution from nylon wool columns (Polysciences). This achieved $>95 \%$ purity without bystander activation of $\mathrm{T}$ cells. 


\section{Plasmids}

An eGFP-containing plasmid, pGEM4Z/eGFP/A64, was obtained from Dr. E. Gilboa, University of Miami. For WT1, an EcoRI insert encoding WT1 cDNA, derived from the pUC119 plasmid (Riken Bioresource), was cloned into a pGEM-4Z vector (Promega). Plasmids were propagated in Max Efficiency DH5- $\alpha$ competent cells (Invitrogen) and purified using a Plasmid Maxi Kit (QIAGEN).

\section{Production of in vitro-transcribed mRNA}

For eGFP mRNA transcription, the pGEM4Z/eGFP/A64 plasmid was linearized with SpeI (New England Biolabs) before mRNA transcription in vitro, which was performed with T7 RNA polymerase (mMessage mMachine T7 kit; Ambion). For WT1 mRNA transcription, the pGEM-4Z/WT1 plasmid was linearized with HindIII (New England Biolabs) before mRNA transcription in vitro, which was performed with SP6 RNA polymerase (mMessage mMachine SP6 kit; Ambion). For both eGFP and WT1 mRNA, agarose gel electrophoresis confirmed production of full-length capped mRNA, and spectrophotometry measured mRNA concentration.

\section{Electroporation of cells}

Immature moDCs were electroporated on day 5-6 and immature LCs on day 10-11. Partially-matured moDCs were electroporated on day 7-8 and partially-matured LCs on day $12-13$, reflecting the different times required to generate these two DC subtypes in vitro $[14,15]$. After harvesting, cells were washed twice and resuspended in OptiMEM (Gibco, Invitrogen) at $15 \times 10^{6}$ cells $/ \mathrm{ml} .200 \mu \mathrm{L}$ of cell suspension were then mixed with $20-40 \mu \mathrm{g}$ mRNA and electroporated in a $2 \mathrm{~mm}$ gap cuvette at 650-900 V for $1-3$ pulses at $0.5 \mathrm{msec} /$ pulse, using a BTX ECM 830 square-wave electroporator (BTX Harvard). After electroporation, cells were immediately transferred to culture to minimize cell death. While in culture, immature cells were partially or terminally matured by exposure to inflammatory cytokines for 24 or 48 hours, respectively.

\section{Phenotypic and eGFP expression analyses by flow cytometry}

Fluorescein (FITC)-, phycoerythrin (PE)-, PE-cyanine -7-, and allophycocyanin (APC)-conjugated mouse anti-human monoclonal antibodies included anti-CD80, anti-CD83, anti-CD86, and anti-human histocompatibility leukocyte antigen (HLA)-DR (BD Biosciences). Nonreactive isotype-matched antibodies (Becton Dickinson) were used as controls. Post-electroporation eGFP expression was assessed, compared with expression by mock-electroporated DCs. Flow cytometry analyses used a Cytomics FC500 flow cytometer (Beckman Coulter) or LSRFortessa cell analyzer (BD Biosciences). Gates were set for collection and analysis of at least 20,000 live events. Data were analyzed with FlowJo software (TreeStar).

\section{Allogeneic mixed leukocyte reactions (MLRs)}

Mock-, eGFP mRNA-, and human WT1 mRNAelectroporated moDCs or LCs were separately added in serial doses (1:10 to 1:1000, moDC:T) to triplicate wells of $1 \times 10^{5}$ allogeneic $\mathrm{T}$ cells in 96 round-bottomed well plates (Corning Life Sciences). Final volume was $100 \mu \mathrm{L} /$ well of complete RPMI-10\% heat-inactivated, NHS serum. Responder allogeneic $\mathrm{T}$ cell proliferation was measured by either: 1) incorporation of methyl-[3H]thymidine $([3 \mathrm{H}] \mathrm{TdR}, 1 \mu \mathrm{Ci} /$ well; New England Nuclear, Division of PerkinElmer Life Sciences) during the last 8 hours of a 5-day culture, as measured in a beta scintillation counter (Betaplate; Wallac, Division of PerkinElmer Life Sciences), or 2) colorimetric assay according to manufacturer's instructions (CellTiter96 Aqueous One Solution Cell Proliferation Assay MTS; Promega).

\section{Statistics}

Student $\mathrm{t}$ test was used for each pairwise comparison. One-way analysis of variance was used for multiple group comparisons. A $P$ value less than .05 was considered statistically significant.

\section{Results}

The transfection efficiency of mRNA electroporation varies with the maturation status of moDCs and LCs Immature and 24-hour, partially-matured moDCs and LCs were electroporated with eGFP mRNA. After electroporation, cells were immediately returned to culture for at least 24 hours of maturation before being assessed for eGFP expression as an index of transfection efficiency. As shown in Figure 1A, transfection efficiency was higher for immature than for partially-matured moDCs (peak value at 24 hours: $77.9 \pm 12.4 \%$ for immature cells and $59.4 \pm 15.4 \%$ for partially-matured cells). In contrast, transfection efficiency was higher for partially-matured than for immature LCs (Figure 1B; peak value at 48 hours: $67 \pm 6.9 \%$ for partially-matured cells and $55.2 \pm 2.9 \%$ for immature cells). Thus, both the type and maturation status of DCs influence mRNA transfection efficiency.

Optimal electroporation parameters for immature moDCs and partially-matured LCs were determined by varying set voltage, number of electroporation pulses, and amount of mRNA to maximize transfection efficiency while minimizing cell loss. For immature moDCs, best results were achieved with $700 \mathrm{~V}, 1$ pulse, and $40 \mu \mathrm{g}$ mRNA. For partially-matured LCs, best results were achieved with 700 V, 2 pulses, and $30 \mu \mathrm{g}$ mRNA. Results are summarized in Figure 2. 

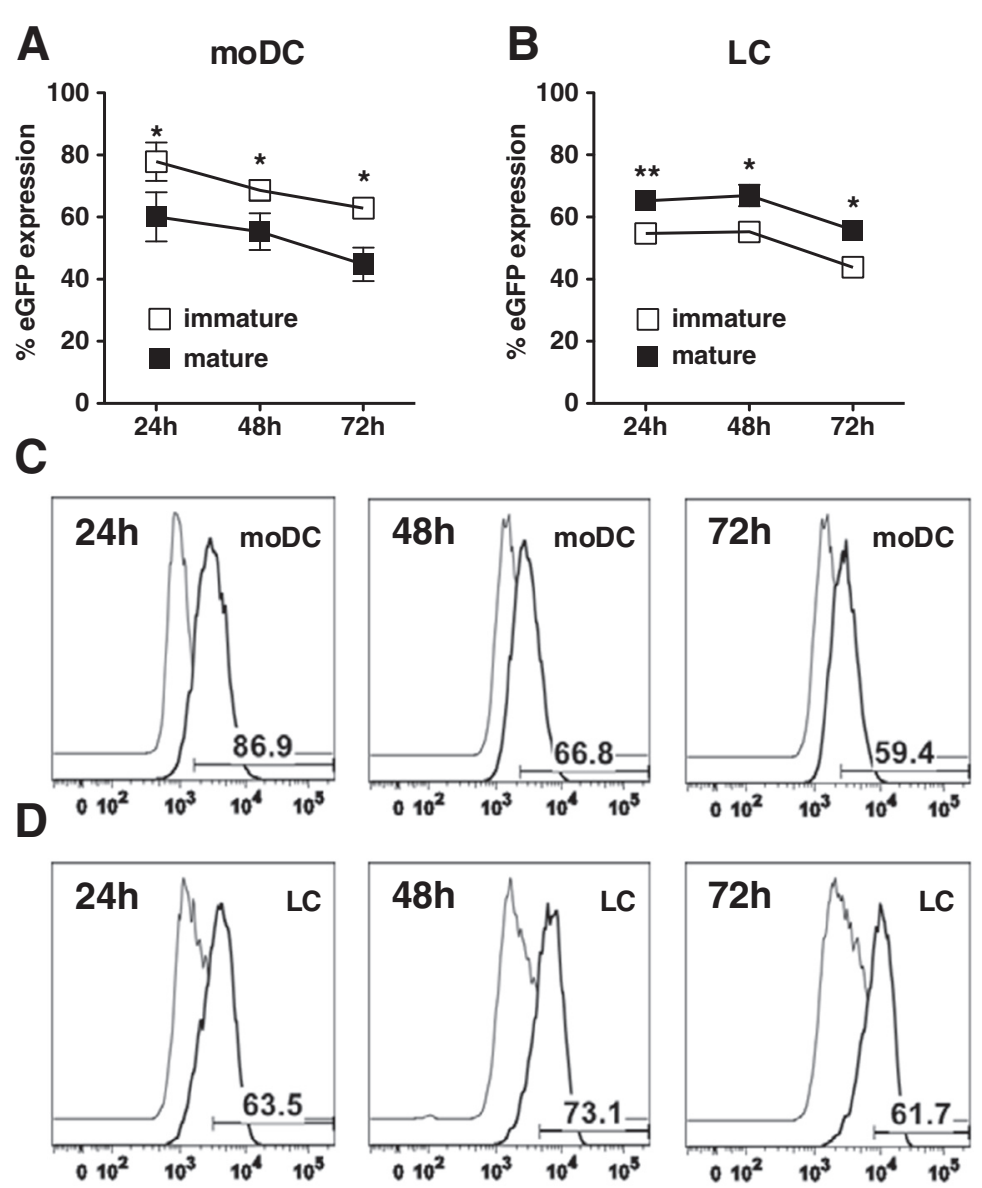

Figure 1 The maturation status of moDCs and LCs affects mRNA-electroporation transfection efficiency. Immature ( $\square$ ) and partiallymatured $(\mathbf{a})$ moDCs (A, C) and LCs (B, D) were electroporated with eGFP-encoding mRNA. The transfection efficiency for each experimental group was assessed by flow cytometry at the indicated time points. Pooled data (mean $\pm S D, n=6$ independent experiments) are shown for moDCs (A) and LCs (B). ${ }^{*} P<.05$ and ${ }^{* *} P<.01$ for comparisons between immature and mature groups. Representative histograms of eGFP mRNA-electroporated immature moDCs (C) and partially-matured LCS (D) from one of six independent experiments are shown.

Immature LCs, in contrast to immature moDCs, display a more mature phenotype after mRNA electroporation Immature moDC and LCs electroporated with eGFP mRNA were incubated with or without standard inflammatory cytokines. After 24 hours, DCs were assessed by flow cytometry for the upregulation of the maturation and activation markers, CD83, CD80, and CD86 [14,15]. Electroporation had a mild direct effect on moDC maturation based on expression of the prototypical DC maturation marker, CD83 (Figure 3A; $10.68 \pm 3.37 \%$ pre-electroporation and $26.13 \pm 3.34 \%$ post-electroporation), whereas electroporation markedly increased the maturation of $\mathrm{CD} 3^{+} \mathrm{HLA}-\mathrm{DR}{ }^{\text {bright }} \mathrm{LCs}$ (Figure 3A; $29.62 \pm 2.8 \%$ pre-electroporation and $96.92 \pm 0.81 \%$ post-electroporation). Electroporation induced greater overall CD80 expression by LCs (Figure 3B; $33.36 \pm$ $1.65 \%$ pre-electroporation and $77.03 \pm 5.54 \%$ postelectroporation) than moDCs (Figure 3B; $4.9 \pm 2.1 \%$ preelectroporation and $35.88 \pm 7.11 \%$ post-electroporation); fold increase of CD80 expression was greater for moDCs (7.3-fold increase for moDC and 2.3-fold increase for LCs). Electroporation had a similar effect on CD86 expression by moDCs (Figure 3C; $37.83 \pm 3.12 \%$ preelectroporation and $73 \pm 5.77 \%$ post-electroporation) and LCs (Figure 3C; $27.44 \pm 2.11 \%$ pre-electroporation and $78.65 \pm 3.12 \%$ post-electroporation). For either DC subtype, electroporation did not impair terminal, inflammatory cytokine-induced maturation.

\section{Cell loss and viability of moDCs and LCs after} electroporation and cryopreservation

Electroporation of cells can induce cell death due to the deleterious effects of direct electrical current and disruption of the cell membrane. Electroporation of immature and partially-matured moDCs and LCs resulted in up to approximately $40 \%$ initial cell death, as determined by trypan blue exclusion on direct hemacytometer counts (Figure 4A). 


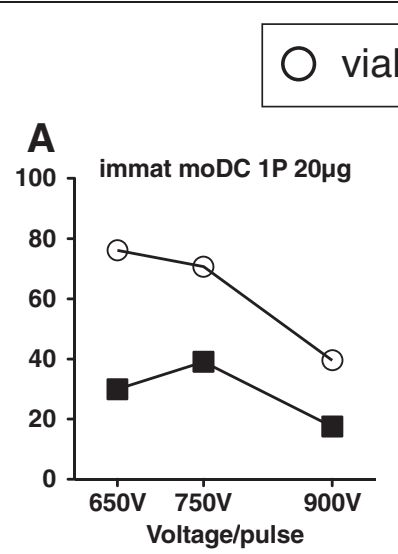

viability

transfection efficiency
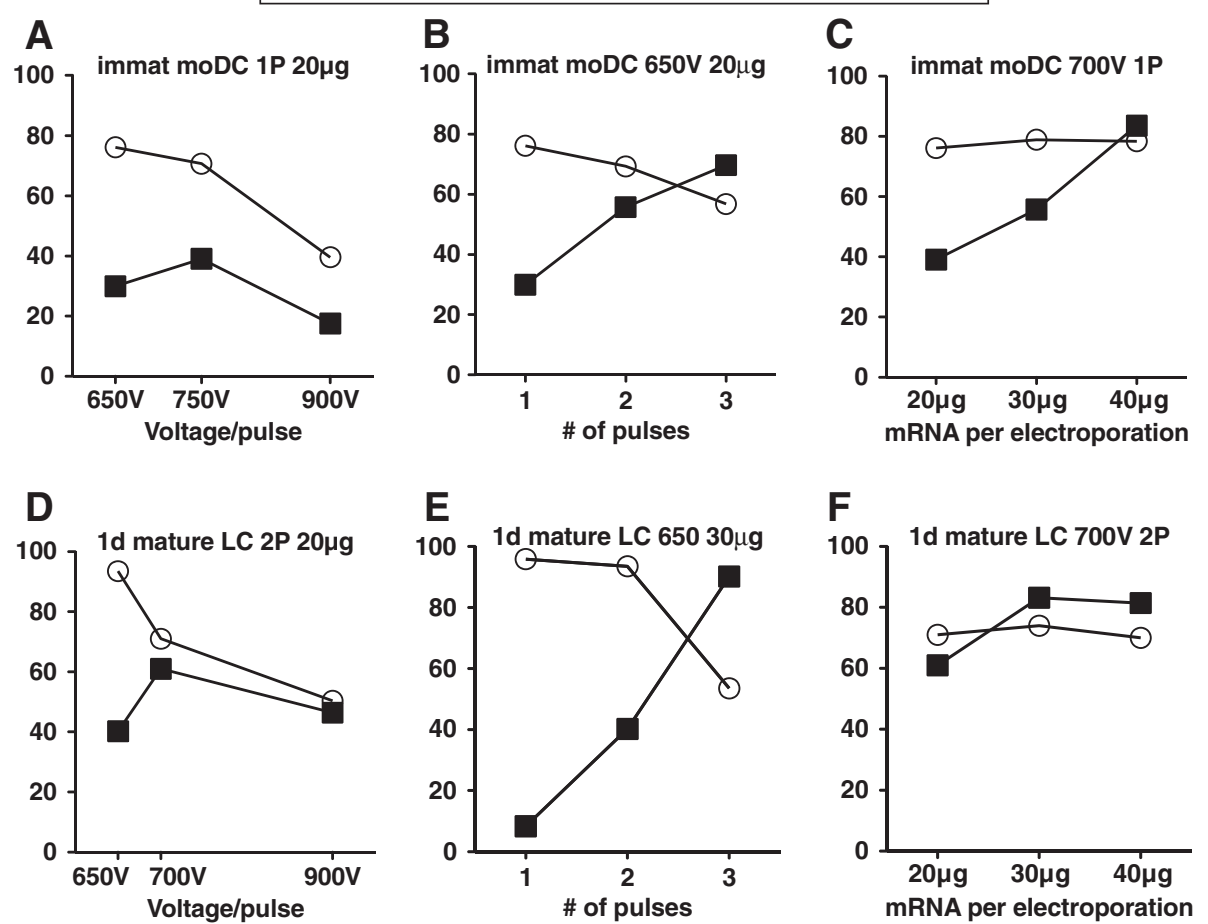

Figure 2 Optimization of mRNA-electroporation conditions. Immature moDCs (A-C) and partially-matured LCs (D-F) were electroporated with eGFP-encoding mRNA under different conditions of set voltage, number of electroporation pulses, or amount of mRNA. Only one of the three parameters was varied in any given set of tested conditions, as summarized in each panel. Cells were assessed for viability ( 0 ) by trypan blue exclusion and transfection efficiency ( $\mathbf{\square}$ ) by flow cytometry.

After electroporation, DCs were returned to culture for 24 hours, and cell viability was reassessed. Immature and mature moDCs and LCs demonstrated $>85 \%$ viability (Figure 4B; $94.2 \pm 3.0 \%$ for immature moDCs and $88.7 \pm 6.3 \%$ for immature LCs; $87.1 \pm 7.1 \%$ for partiallymatured moDCs and $92.9 \pm 3.8 \%$ for partially-matured LCs).

The viability of both DC subtypes was further evaluated after cryopreservation, with no significant difference in survival between moDCs and LCs immediately after thawing (Figure 4C; $79.2 \pm 9.8 \%$ for immature moDCs and $83.6 \pm 5.4 \%$ for immature LCs; $82.3 \pm 5.2 \%$ for partially-matured moDCs and $84.4 \pm 5.4 \%$ for partiallymatured LCs) or after thawing and resting for 24 hours (Figure 4D; $96.8 \pm 2.7 \%$ for immature moDCs and $94.8 \pm$ $6.7 \%$ for immature LCs; $95.5 \pm 8.4 \%$ for partially-matured moDCs and $94.9 \pm 4.2 \%$ for partially-matured LCs). These anticipated yields are reproducible and inform the required number of starting cells.

\section{MoDCs and LCs electroporated with mRNA retain} transgene expression after cryopreservation Immature and mature moDCs and LCs were electroporated with eGFP mRNA, and post-electroporation
eGFP expression was determined by flow cytometry. Cells were then cryopreserved, and post-thaw eGFP expression was compared with expression prior to cryopreservation. Retention of protein expression was similar between immature and mature moDCs and LCs (Figure 5).

\section{mRNA-electroporated moDCs and LCs remain potent} stimulators of allogeneic $\mathrm{T}$ cells in mixed leukocyte reactions

Having shown that mRNA-electroporated moDCs and LCs possess the ability to respond normally to inflammatory cytokines by upregulating phenotypic markers of maturation and activation, we further assessed their functional capacity to stimulate resting allogeneic $\mathrm{T}$ cell proliferation. While distinct DC subtypes have different capacities to stimulate $\mathrm{CD}^{+}$CTLs $[14,15]$, their comparable stimulation of bulk allogeneic $\mathrm{T}$ cell proliferation in MLRs remains a standard assay of overall DC immunogenicity [14]. MoDCs and LCs were therefore electroporated with eGFP or WT1 mRNA, or were mock electroporated with no mRNA and then matured with inflammatory cytokines for 24 hours. The resulting mature moDCs (Figure 6A, left panel) or LCs (Figure 6A, 

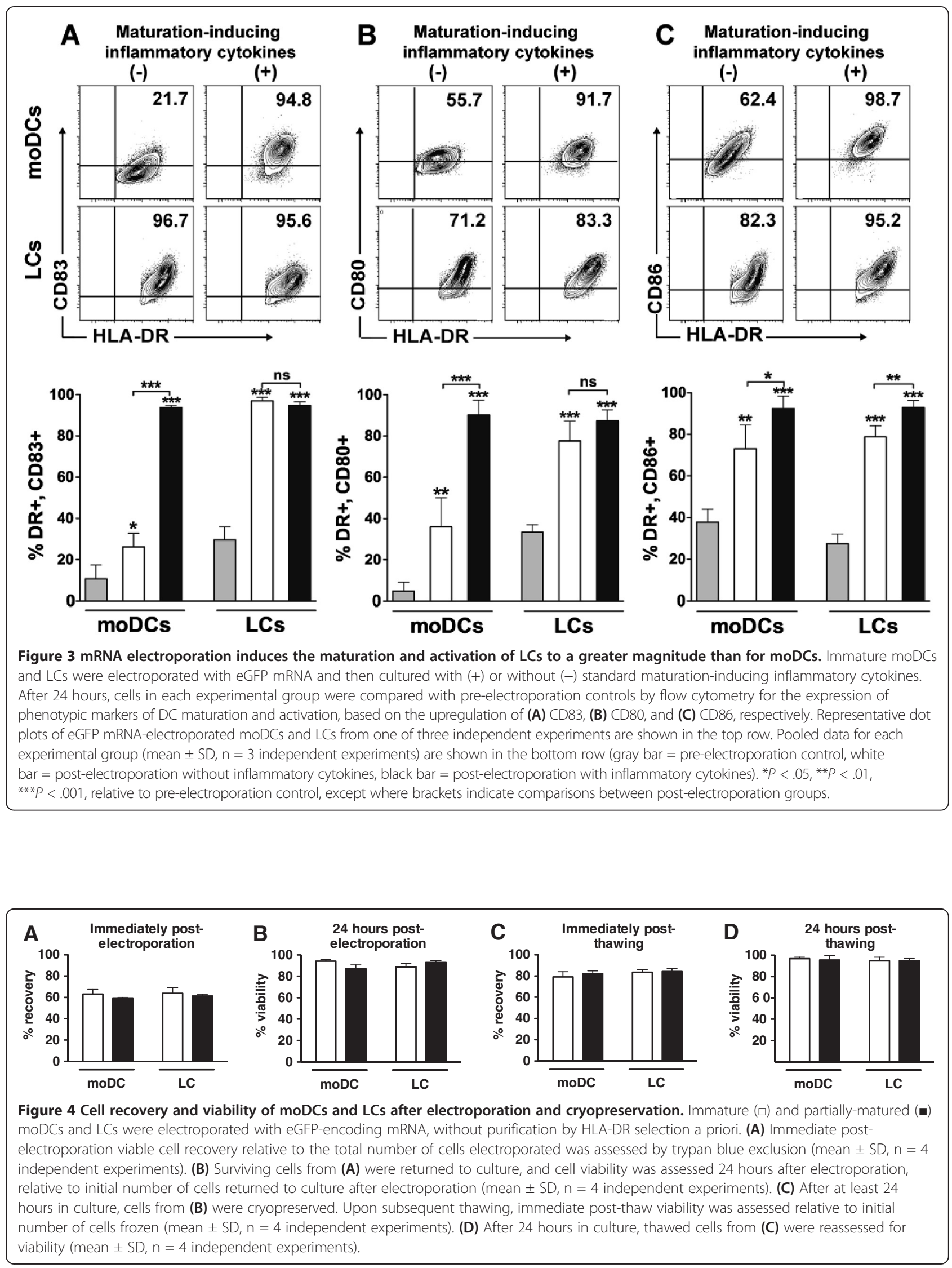


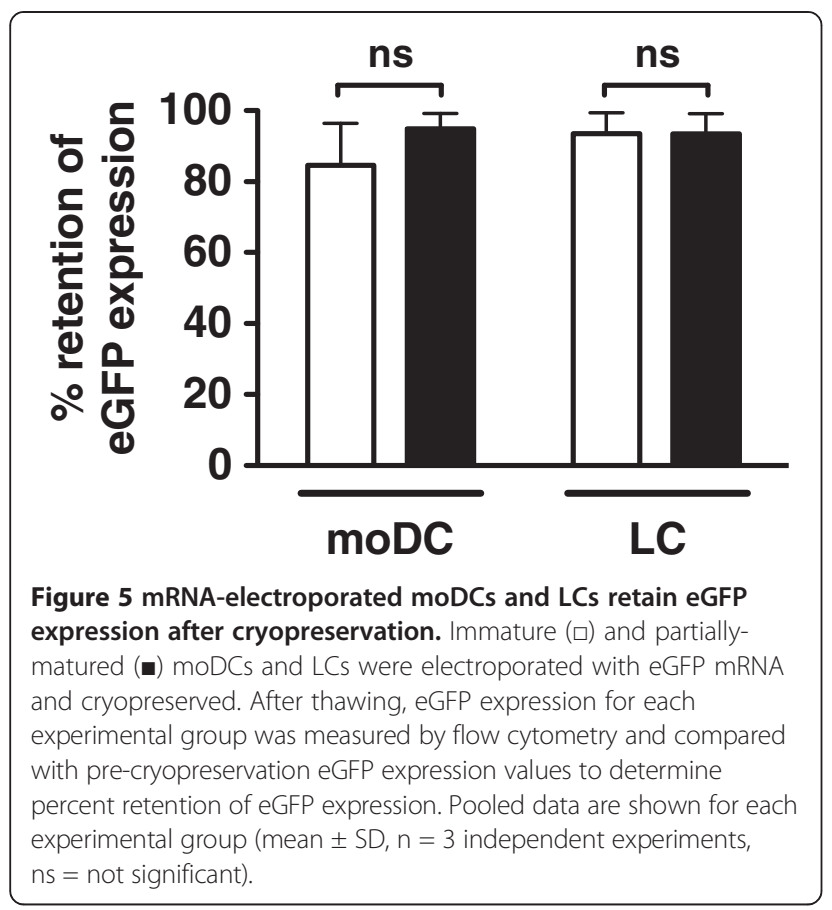

right panel) were added in serial 3-fold dilutions to a fixed number of purified allogeneic $\mathrm{T}$ cells and incubated for 5 days. Allogeneic T cell proliferative responses were measured by $[3 \mathrm{H}] \mathrm{TdR}$ uptake over the last 8 hours of a 5 -day culture. Responder T cells showed similar trends of proliferation when cultured with moDC and LCs electroporated under the three experimental conditions (Figure 6A). DCs also retained their allo-stimulatory capacity after cryopreservation and thawing, as measured by a colorimetric proliferation assay (Figure 6B). Thus, both moDCs and LCs remain functionally potent stimulators of allogeneic $\mathrm{T}$ cell proliferation, unaffected by prior mRNA electroporation or cryopreservation and despite their differences in CTL stimulation.

\section{Discussion}

This study establishes different conditions for the successful electroporation and expression of full-length mRNA in human CD34 ${ }^{+}$HPC-derived LCs versus blood moDCs, for use in clinical DC-based vaccine trials. Electroporation efficiency depends on the maturation status of the two DC subtypes, with partially-matured LCs showing more efficient transfection than immature LCs and immature moDCs showing more efficient transfection than mature moDCs. Electroporation favorably induces the maturation of CD83 ${ }^{+} \mathrm{HLA}^{-D R^{\text {bright }}} \mathrm{LCs}$, whereas electroporation has no direct effect on moDC maturation. LCs and moDCs are equally sensitive to the direct effects of electroporation with decreased viability immediately after electroporation. LCs and moDCs also demonstrate similar retention of transgene expression after cryopreservation. Importantly, both DC subtypes maintain potent immunogenicity after electroporation

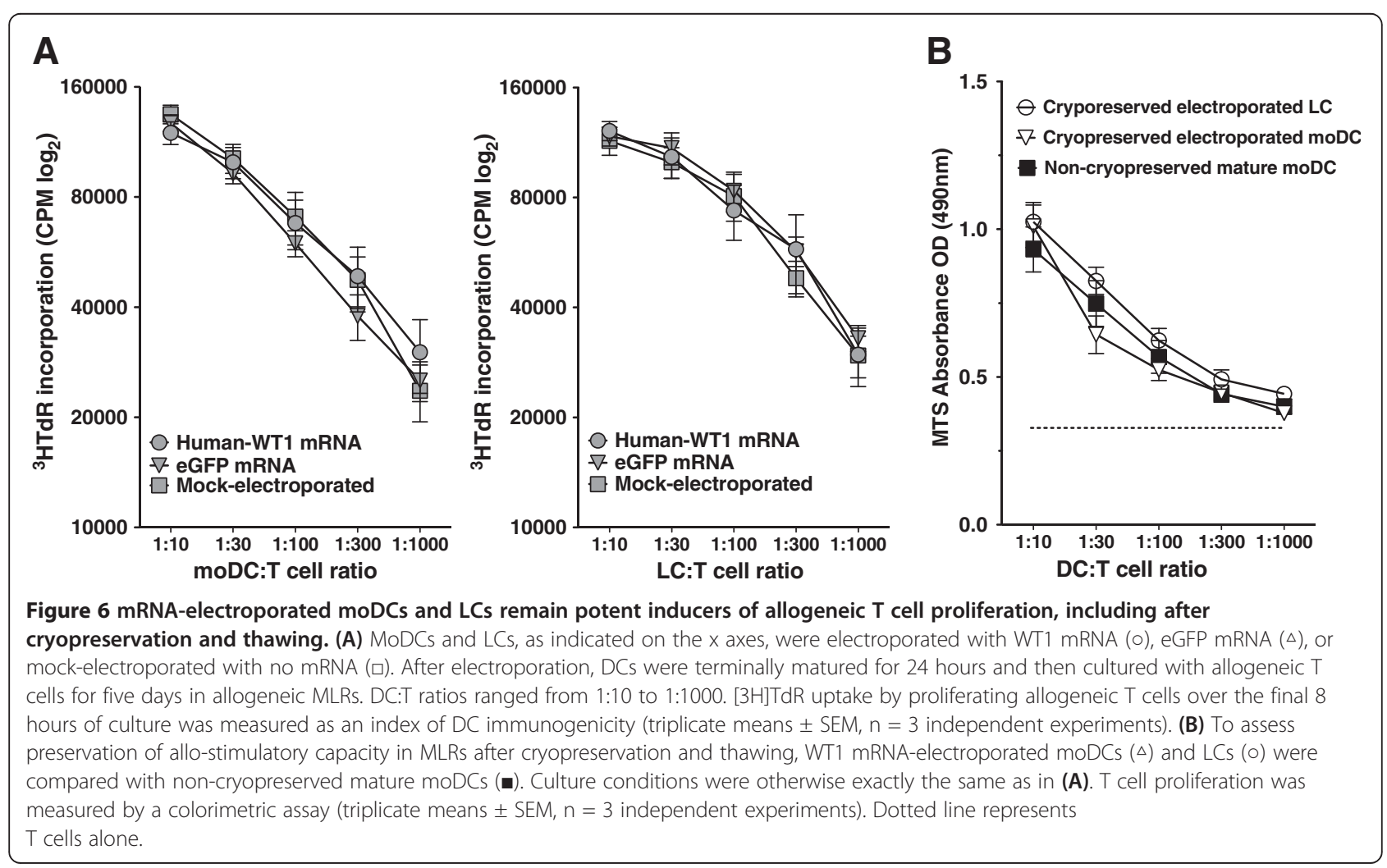


for stimulating allogeneic $\mathrm{T}$ cell proliferation in MLRs, despite their inherent differences in capacity for $\mathrm{CD}^{+}$ CTL stimulation [14-17].

For moDCs, our observation of greater transfection efficiency of immature cells than for mature cells differs from another study in which the transfection efficiency of immature and mature moDCs was roughly equivalent [18]. Various electroporation conditions with disparate degrees of transfection efficiency, however, have been reported for moDCs $[8,19-21]$. These discrepancies underscore the importance of revalidating electroporation parameters for any differences in electroporation machine, voltage, number of pulses, cell concentration, or amount of mRNA, as depicted in Figure 2.

Peak transfection efficiency is lower for LCs than for moDCs. Likely contributing to this difference is that moDCs are derived from a more differentiated and committed starting population than are LCs, resulting in greater cell population purity. Thus the difference in absolute transfection values would have favored moDCs, as the LCs would have had other contaminating, non-LCs competing for mRNA uptake during electroporation. Purification of LCs by HLA-DR selection on magnetic beads is one potential solution to increase transfection efficiency, but this should not be necessary to the extent that one can quantify the proportion of electroporated LCs by flow cytometry in the final antigen-presenting cell population.

Consistent with previous studies [22-24], the immediate post-electroporation viability of mRNA-electroporated DCs is less than the initial starting population. Subsequent survival of viable cells is minimally affected, however. Cryopreservation can further decrease cell recovery, so an appropriate excess of electroporated DCs should be aliquoted for cryopreservation. This will ensure sufficient DC yields, irrespective of moDC or LC subtype, from thawed aliquots for vaccination.

We previously reported the results of a clinical trial in AJCC stage III/IV melanoma patients, comparing vaccination with peptide-loaded LCs versus peptide-loaded moDCs [12]. The rationale for this trial was based on our established findings in vitro that human LCs are consistently superior to moDCs on a cell-for-cell basis in eliciting MHC-restricted, antigen-specific CTLs $[14,16]$, which has been corroborated by other investigators [15,17]. A clinical trial conducted by Banchereau and colleagues for similarly advanced-stage melanoma patients using bulk CD34 ${ }^{+}$HPC-derived DCs, which included LCs, pulsed with a mixture of melanoma-derived peptides, resulted in durable immune responses associated with long-term survival [25]. Although patients treated on the LC arm of our trial generated significantly greater reactivity against tyrosinase than those treated with moDCs, significant differences were not observed for gp100 or the control fluMP antigen [12]. This begged the obvious question as to whether loading class I MHC-restricted single peptides onto any defined DC subtype would ever be sufficient to stimulate durable immunity against tumor antigens.

Our group has since discovered that LCs from healthy volunteers, electroporated with Wilms' tumor 1 (WT1) mRNA, promote sustained presentation of antigenic peptides, which in conjunction with IL15R- $\alpha /$ IL15, induce robust autologous, WT1-specific CTLs [16]. The CTLs develop after just 7 days' stimulation without exogenous cytokine supplementation and lyse MHCrestricted targets, including primary WT1-expressing blasts from leukemia patients. MoDCs, in contrast, require exogenous IL15 to promote immune responses comparable to LCs. Thus LCs provide a more favorable cytokine milieu for the activation of $\mathrm{T}$ cells than do moDCs, thereby explaining their superior induction of antigen-specific CTLs in the absence of exogenous cytokines. These data support the use of mRNA-electroporated LCs, or IL15-supplemented moDCs, as cancer vaccines to overcome tolerance against self-differentiation tumor antigens. The pivotal role of IL15 in LCmediated stimulation of CTLs has also been confirmed using individual peptides rather than mRNA encoding full-length protein $[17,25]$.

Given the importance of LCs and their provision of IL15, as well as the possible application of IL15supplemented moDCs in DC-based vaccines, we thought it essential to ascertain the optimal conditions for their respective electroporation with mRNA to express fulllength protein. Such expression enables cells to process and present antigenic peptides tailored to their own MHCs, allowing clinical investigators to move beyond single defined peptides for a given tumor, if they are even known, and circumvent the limitations of specific HLA types in patients. There may be additional, as yet undiscovered reasons for LCs' potency over moDCs, which may nonetheless prove sufficiently compelling to favor LCs in vaccine trials. MoDCs offer the potential benefit of activating NK cells via IL12p70 [26]. LCs do not secrete IL12p70 but maintain NK cell viability via IL15 $[14,26]$. It will be important to determine whether moDCs supplemented with IL15 are an adequate substitute for LCs, or whether the two DC subtypes should be used in combination to capitalize on the efficacy of moDCs in activating NK cells and the potency of LCs in inducing $\mathrm{CD} 8^{+}$CTLs.

\section{Conclusions}

Enhanced antigen presentation by DCs can be achieved with mRNA electroporation but requires modifying conditions to the specific DC subtype. Our findings with LCs and moDCs provide key parameters on the optimal 
timing of mRNA electroporation, effects on cell maturation, and anticipated cell losses and yields. Investigators should now have the tools in hand to address key questions about DC-based cancer immunotherapy in vivo in humans, with reasonable maintenance of LC or moDC viability and preservation of the activated phenotype and baseline immunostimulatory capacity.

\section{Abbreviations}

APC: Antigen-presenting cell; CTL: Cytotoxic T lymphocyte; DC: Dendritic cell; eGFP: Enhanced green fluorescent protein; FLT-3-ligand: Fms-related tyrosine kinase-3-ligand; fluMP: Flu matrix peptide; G-CSF: Granuloctye colonystimulating factor; GM-CSF: Granuloctye macrophage colony-stimulating factor; HPC: Hematopoietic progenitor cell; IL-1 $\beta$ : Interleukin-1-beta; IL-6: Interleukin-6; IL12p70: Interleukin-12p70; IL15: Interleukin-15; IL15Ralpha: Interleukin-15-receptor-alpha; LC: Langerhans-type DC; MHC: Major histocompatibility complex; MLR: Mixed leukocyte reaction; moDC: Monocyte-derived DC; NHS: Normal human serum; NK cell: Natural killer cell; pSTAT5: Phosphorylated signal transducer and activator of transcription 5; TGF- $\beta$ : Transforming growth factor-beta; TNF-a: Tumor necrosis factor-alpha; WT1: Wilms' tumor 1.

\section{Competing interests}

The authors have no competing interests to declare.

\section{Authors' contributions}

DJC designed and performed experiments, analyzed and interpreted data and wrote the manuscript. ER designed and performed experiments, analyzed and interpreted data, and made comments on the manuscript. ER and MM designed the WT1-encoding plasmid. KBP, JAS, and ETSA performed experiments. JWY designed the overall study, supervised performance of experiments, analyzed and interpreted data, and wrote the manuscript. All authors read and approved the final manuscript.

\section{Acknowledgments}

We gratefully acknowledge the various contributions of members of the Laboratory of Cellular Immunobiology to the development of this work. We thank the nurses and physicians of the Allogeneic Bone Marrow Transplantation Service, as well as the Blood Donor Room and Cytotherapy Laboratory staffs at MSKCC, for assistance with sample procurement and processing. We also thank the patients and healthy volunteers who provided samples for research.

\section{Funding support}

CA100463 (DJC) from the Congressionally Directed Medical Research Programs, Department of Defense. R01-CA083070 (JWY), R01-CA118974 (JWY), R21-CA119528 (JWY), P01-CA23766 (GH, JWY), from the National Cancer Institute, National Institutes of Health; Mr. William H. Goodwin and Mrs. Alice Goodwin of the Commonwealth Cancer Foundation for Research and The Experimental Therapeutics Center of Memorial Sloan-Kettering Cancer Center (JWY); and Swim Across America (JWY).

\section{Author details}

'Laboratory of Cellular Immunobiology, New York, NY, USA. ${ }^{2}$ Swim Across America Laboratory, New York, NY, USA. ${ }^{3}$ Adult Bone Marrow Transplant Service, Division of Hematologic Oncology, New York, NY, USA. ${ }^{4}$ Department of Medicine, New York, NY, USA. ${ }^{5}$ Immunology Program, Sloan-Kettering Institute for Cancer Research, New York, NY, USA. ${ }^{6}$ Memorial Sloan-Kettering Cancer Center, New York, NY, USA. 'Weill Cornell Medical College, New York, NY, USA. ${ }^{8}$ Centre Hospitalier Universitaire Vaudois, CHUV-BH06, Rue du Bugnon 46, 1011, Lausanne, Switzerland/Suisse. ${ }^{9}$ European Commission, Joint Research Centre, Ispra (VA) 21027, Italy.

Received: 19 December 2012 Accepted: 27 June 2013 Published: 9 July 2013

\section{References}

1. Guermonprez P, Valladeau J, Zitvogel L, Thery C, Amigorena S: Antigen presentation and T cell stimulation by dendritic cells. Annu Rev Immunol 2002, 20:621-667.

2. O'Neill DW, Adams S, Bhardwaj N: Manipulating dendritic cell biology for the active immunotherapy of cancer. Blood 2004, 104:2235-2246.

3. Palucka K, Banchereau J: Cancer immunotherapy via dendritic cells. Nat Rev Cancer 2012, 12:265-277.

4. Nair SK, Boczkowski D, Morse M, Cumming Rl, Lyerly HK, Gilboa E: Induction of primary carcinoembryonic antigen (CEA)-specific cytotoxic $T$ lymphocytes in vitro using human dendritic cells transfected with RNA. NatBiotech 1998, 16:364-369.

5. Heiser A, Dahm P, Yancey DR, Maurice MA, Boczkowski D, Nair SK, Gilboa E, Vieweg J: Human dendritic cells transfected with RNA encoding prostate-specific antigen stimulate prostate-specific CTL responses in vitro. J Immunol 2000, 164:5508-5514.

6. Heiser A, Maurice MA, Yancey DR, Coleman DM, Dahm P, Vieweg J: Human dendritic cells transfected with renal tumor RNA stimulate polyclonal $\mathrm{T}$-cell responses against antigens expressed by primary and metastatic tumors. Cancer Res 2001, 61:3388-3393.

7. Heiser A, Coleman D, Dannull J, Yancey D, Maurice MA, Lallas CD, Dahm P, Niedzwiecki D, Gilboa E, Vieweg J: Autologous dendritic cells transfected with prostate-specific antigen RNA stimulate CTL responses against metastatic prostate tumors. J Clin Invest 2002, 109:409-417.

8. Van Tendeloo VF, Ponsaerts P, Lardon F, Nijs G, Lenjou M, Van Broeckhoven C, Van Bockstaele DR, Berneman ZN: Highly efficient gene delivery by mRNA electroporation in human hematopoietic cells: superiority to lipofection and passive pulsing of mRNA and to electroporation of plasmid CDNA for tumor antigen loading of dendritic cells. Blood 2001, 98:49-56.

9. Tuyaerts S, Michiels A, Corthals J, Bonehill A, Heirman C, de Greef C, Noppe SM, Thielemans K: Induction of Influenza Matrix Protein 1 and MelanA-specific T lymphocytes in vitro using mRNA-electroporated dendritic cells. Cancer Gene Ther 2003, 10:696-706.

10. Milazzo C, Reichardt VL, Muller MR, Grunebach F, Brossart P: Induction of myeloma-specific cytotoxic $T$ cells using dendritic cells transfected with tumor-derived RNA. Blood 2003, 101:977-982.

11. Van Tendeloo VF, Van de Velde A, Van Driessche A, Cools N, Anguille S, Ladell K, Gostick E, Vermeulen K, Pieters K, Nijs G, et al: Induction of complete and molecular remissions in acute myeloid leukemia by Wilms' tumor 1 antigen-targeted dendritic cell vaccination. Proc Natl Acad Sci U S A 2010, 107:13824-13829.

12. Romano E, Rossi M, Ratzinger $\mathrm{G}$, de Cos MA, Chung DJ, Panageas KS, Wolchock JD, Houghton AN, Chapman PB, Heller G, et al: Peptide-Loaded Langerhans Cells, Despite Increased IL15 Secretion and T-Cell Activation In Vitro, Elicit Antitumor T-Cell Responses Comparable to PeptideLoaded Monocyte-Derived Dendritic Cells In Vivo. Clin Cancer Res 2011, 17:1984-1997.

13. Gilboa E, Vieweg J: Cancer immunotherapy with mRNA-transfected dendritic cells. Immunol Rev 2004, 199:251-263.

14. Ratzinger G, Baggers J, de Cos MA, Yuan J, Dao T, Reagan JL, Munz C, Heller G, Young JW: Mature human Langerhans cells derived from CD34+ hematopoietic progenitors stimulate greater cytolytic T lymphocyte activity in the absence of bioactive IL-12p70, by either single peptide presentation or cross-priming, than do dermal-interstitial or monocytederived dendritic. cells J Immunol 2004, 173:2780-2791. Erratum in J Immunol. 2005; 2174:3818.

15. Klechevsky E, Morita R, Liu M, Cao Y, Coquery S, Thompson-Snipes L, Briere F, Chaussabel D, Zurawski G, Palucka AK, et al: Functional specializations of human epidermal Langerhans cells and CD14+ dermal dendritic cells. Immunity 2008, 29:497-510.

16. Romano E, Cotari JW, Barreira DaSilva R, Betts BC, Chung DJ, Avogadri F, Fink MJ, St Angelo ET, Mehrara B, Heller G, et al: Human Langerhans cells use an IL-15R-alpha/L-15/pSTAT5-dependent mechanism to break T-cell tolerance against the self-differentiation tumor antigen WT1. Blood 2012, 119:5182-5190.

17. Banchereau J, Thompson-Snipes L, Zurawski S, Blanck J-P, Cao Y, Clayton S, Gorvel J-P, Zurawski G, Klechevsky E: The differential production of cytokines by human Langerhans cells and dermal CD14+ DCs controls CTL priming. Blood 2012, 119:5742-5749. 
18. Michiels A, Tuyaerts S, Bonehill A, Corthals J, Breckpot K, Heirman C, Van Meirvenne S, Dullaers M, Allard S, Brasseur F, et al: Electroporation of immature and mature dendritic cells: implications for dendritic cell-based vaccines. Gene Ther 2005, 12:772-782

19. Jarnjak-Jankovic S, Pettersen RD, Saeboe-Larssen S, Wesenberg F, Gaudernack G: Evaluation of dendritic cells loaded with apoptotic cancer cells or expressing tumour mRNA as potential cancer vaccines against leukemia. BMC Cancer 2005, 5:20.

20. Van den Bosch GA, Ponsaerts P, Nijs G, Lenjou M, Vanham G, Van Bockstaele DR, Berneman ZN, Van Tendeloo VF: Ex vivo induction of viral antigenspecific CD8 T cell responses using mRNA-electroporated CD40activated B cells. Clin Exp Immunol 2005, 139:458-467.

21. Strobel I, Berchtold S, Gotze A, Schulze U, Schuler G, Steinkasserer A: Human dendritic cells transfected with either RNA or DNA encoding influenza matrix protein $\mathrm{M} 1$ differ in their ability to stimulate cytotoxic T lymphocytes. Gene Ther 2000, 7:2028-2035.

22. Van Driessche A, Van de Velde AL, Nijs G, Braeckman T, Stein B, De Vries JM, Berneman ZN, Van Tendeloo VF: Clinical-grade manufacturing of autologous mature mRNA-electroporated dendritic cells and safety testing in acute myeloid leukemia patients in a phase I dose-escalation clinical trial. Cytotherapy 2009, 11:653-668.

23. Markovic SN, Dietz AB, Greiner CW, Maas ML, Butler GW, Padley DJ, Bulur $P A$, Allred JB, Creagan ET, Ingle JN, et al: Preparing clinical-grade myeloid dendritic cells by electroporation-mediated transfection of in vitro amplified tumor-derived mRNA and safety testing in stage IV malignant melanoma. J Trans/ Med 2006, 4:35.

24. Dietz AB, Padley DJ, Butler GW, Maas ML, Greiner CW, Gastineau DA, VukPavlovic S: Clinical-grade manufacturing of DC from CD14+ precursors: experience from phase I clinical trials in CML and malignant melanoma. Cytotherapy 2004, 6:563-570.

25. Banchereau J, Palucka AK, Dhodapkar M, Burkeholder S, Taquet N, Rolland A, Taquet S, Coquery S, Wittkowski KM, Bhardwaj N, et al: Immune and clinical responses in patients with metastatic melanoma to CD34(+) progenitorderived dendritic cell vaccine. Cancer Res 2001, 61:6451-6458.

26. Munz C, Dao T, Ferlazzo G, de Cos MA, Goodman K, Young JW: Mature myeloid dendritic cell subsets have distinct roles for activation and viability of circulating human natural killer cells. Blood 2005, 105:266-273.

doi:10.1186/1479-5876-11-166

Cite this article as: Chung et al.: Langerhans-type and monocyte-derived human dendritic cells have different susceptibilities to mRNA

electroporation with distinct effects on maturation and activation:

implications for immunogenicity in dendritic cell-based

immunotherapy. Journal of Translational Medicine 2013 11:166.

\section{Submit your next manuscript to BioMed Central and take full advantage of:}

- Convenient online submission

- Thorough peer review

- No space constraints or color figure charges

- Immediate publication on acceptance

- Inclusion in PubMed, CAS, Scopus and Google Scholar

- Research which is freely available for redistribution 